\title{
Correction for window transmission losses in radiation thermometry using high temperature fixed points
}

\author{
David Lowe ${ }^{1}$, Frédéric Bourson ${ }^{3}$, Christophe Journeau $^{2}$, Graham Machin $^{1}$, Clemente Parga ${ }^{2}$ and Mohamed Sadli ${ }^{3}$ \\ ${ }^{1}$ National Physical Laboratory, Hampton Road, Teddington TW11 0LW, UK \\ ${ }^{2}$ CEA, DEN, Cadarache, STRI/LMA, 13108 St Paul lez Durance, France \\ ${ }^{3}$ Laboratoire Commun de Métrologie (LNE-Cnam), Saint-Denis, France
}

\begin{abstract}
Monitoring processes taking place inside furnaces often involves measurement of temperature using a radiation thermometer looking through a window of unknown, and possibly varying, transmission. This can introduce significant errors. In this paper we consider two approaches to dealing with this problem based on a particular case using a vacuum induction furnace. The use of a ratio radiation thermometer is compared to measurements of a known reference point embedded in the process using a monochromatic radiation thermometer to generate a correction curve. It is found that an included reference can give a correction with minimal residual uncertainty.
\end{abstract}

Les processus de suivi qui se déroulent à l'intérieur des fours implique souvent la mesure de température à l'aide d'un pyromètre en regardant à travers une fenêtre de l'inconnu, et peut-être variable, transmission. Cela peut introduire des erreurs importantes. Dans cet article, nous considérons deux approches pour traiter ce problème sur la base d'un cas particulier en utilisant un four à induction sous vide. L'utilisation d'un pyromètre à rapport est comparé à des mesures d'un point de référence connu incorporé dans le processus en utilisant une seule longueur d'onde de pyromètre pour générer une courbe de correction. Il se trouve qu'une référence inclus peut donner une correction avec un minimum d'incertitude résiduelle.

\section{Introduction}

High temperature fixed points (up to 3000 kelvin) [1] can be used as in-situ reference artefacts [2]. Suitably designed they are extremely robust and can withstand repeated rapid temperature changes [3]. This means it is possible to use a reference point to calibrate a radiation thermometer in-situ, thus taking account of unknown window transmission losses.

The difficulty of dealing with transmission losses is well known and there are different approaches. One is to use a ratio radiation thermometer. In this case any transmission loss is assumed to be the same at two wavelengths $\left(\lambda_{1}\right.$ and $\left.\lambda_{2}\right)$ and so the ratio is only a function of source radiance. As such if everything behaves as a greybody, a temperature can be determined regardless of the window transmission (and source emissivity providing it is grey in the operating wavelength range of the pyrometer). In practice the uncertainties of a ratio pyrometer scale as $\left(1 / \lambda_{1}-1 / \lambda_{2}\right)^{-1}$ and so are always larger than for a monochromatic pyrometer [4]. The problem arises that if the two wavelengths are close together the uncertainties increase rapidly, if they are far apart the assumption of greybody conditions is less likely to be valid. Under some circumstances the advantages may outweigh the increased uncertainties, but this should be assessed case by case.

An alternative to using a ratio radiation thermometer is to use an in situ reference fixed point to calibrate a monochromatic radiation thermometer in combination with the window.

In this study we consider both approaches, based on measurements made during, and subsequent to, trials on the use of high temperature fixed points in the VITI research furnace at CEA, Cadarache [5]. In that study [3] measurements were made of high temperature fixed points (cobalt-carbon, ruthenium-carbon and rheniumcarbon) within a vacuum induction furnace, to assess the practicality of potentially fragile artefacts under rapid temperature changes. The opportunity was taken to make additional measurements with a spectrometer 
as well as with a calibrated radiation thermometer. To calibrate the spectrometer, a ruthenium-carbon fixed point with a known melting temperature was measured through the furnace window. This allowed the spectrometer/window combination to be calibrated. The ratio of any two wavelengths could then be used to determine a temperature and the variation in temperature with the choice of wavelengths.

One issue that needs to be considered with radiation thermometers, even ones that work at a single wavelength is that of bandwidth. The measured signal from a radiation thermometer other than one with an ideal monochromatic response depends both on the thermometer response and the emitted spectrum of the object measured. Typically, because of the shape of the Planck curve, this biases the response of the radiation thermometer to longer wavelengths at higher temperatures. Therefore although the window has fixed transmission loss, the effect on temperature cannot be determined by a single fixed-point measurement.

The temperature dependent wavelength $\lambda_{\mathrm{T}}$ can be written as a series [5]

$$
\lambda_{T}=a_{1}+\frac{a_{2}}{T}+\frac{a_{3}}{T^{2}}+\cdots
$$

where $a_{1}, a_{2}, a_{3} \ldots$ are constants that depend on the central wavelength $\left(\lambda_{0}\right)$ and bandpass width $(\sigma)$ of the radiation thermometer spectral response. A further constant $\mathrm{a}_{0}$ is needed to specify the overall sensitivity of the thermometer. A purely monochromatic device can be specified by Planck's radiation law with two measurements (to determine $\mathrm{a}_{0}: \mathrm{a}_{1}$ ), a narrow band device with three measurements (to determine $\mathrm{a}_{0}: . \mathrm{a}_{2}$ ) and a broadband device with four measurements (to determine $\left.\mathrm{a}_{0}: \mathrm{a}_{3}\right)$.

In the present study we investigated the simplest scheme for correcting a radiation thermometer with direct temperature readout. It was assumed that the instruments linearization approximated the Planck function. This allows estimation of an effective wavelength by reducing radiance a known amount and observing the change in indicated temperature, or equivalently using the emissivity control to interrogate the instrument [6]. On the assumption that $\mathrm{a}_{3}$ and higher terms could be neglected, then given a value for $\sigma$ only one wavelength $\lambda_{0}$ will give the correct change in temperature. With values for $\lambda_{0}$ and $\sigma, a_{1}$ and $a_{2}$ can be calculated. Finally, by measuring the reduction in radiance temperature of a known reference due to the effect of the window, $\mathrm{a}_{0}$ can be determined and so a general correction curve can be established. By varying $\sigma$ between reasonable limits the sensitivity to choice of assumed filter shape can be evaluated.

We compare how the uncertainty of the spectrometer approach and the single wavelength with fixed point corrections compare.
Small fixed-points of cobalt-carbon, ruthenium-carbon and rhenium-carbon were placed in a vacuum induction furnace and measurements made as described in [2]. The melt and freeze transitions were measured through a window of unknown transmission.

\subsection{Spectrometer measurements}

A Hamamatsu spectrometer with a fibre optic coupling was connected to a lens to give a field of view of $1 \mathrm{~mm}$ at a distance of $600 \mathrm{~mm}$. This was set up viewing through the furnace window and aligned on the cavity aperture of the ruthenium-carbon fixed-point by viewing between the induction coil rings and through a hole in the susceptor (Figure 1).
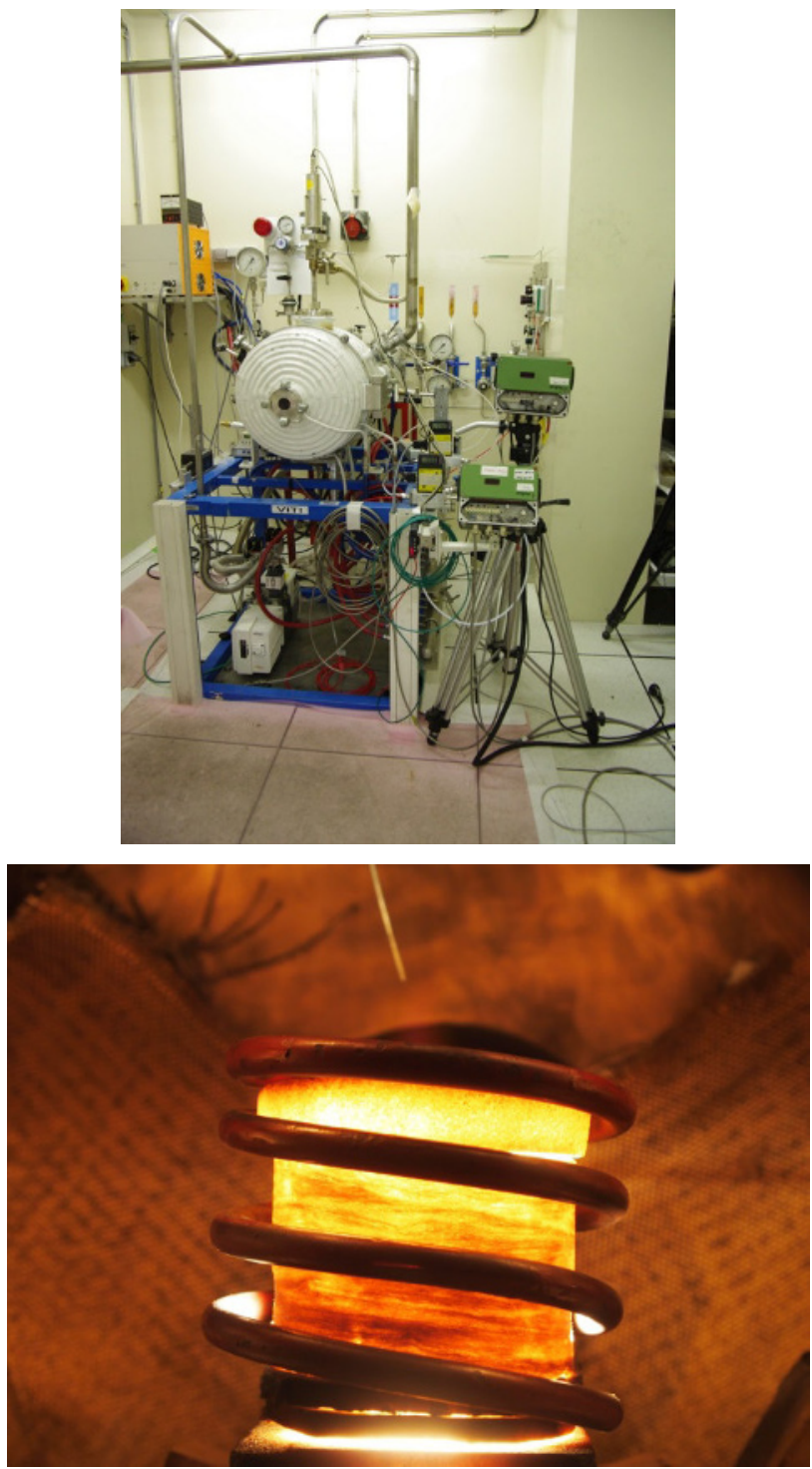

Figure 1.The VITI furnace. Top: general view of the facility. Bottom: heated graphite insulation. The fixed point is viewed from the side, viewing between the induction coils in the VITI furnace through a glass window.

The spectrometer covers wavelengths from $400 \mathrm{~nm}$ to $1100 \mathrm{~nm}$. It was set to an integration time of $100 \mathrm{~ms}$, and 50 readings were averaged to give a full spectrum every 5 secondss. The spectrometer was logged during

\section{Method}


the melt and freeze cycles that were initiated by varying the power to the induction coil. Since the temperature of the fixed point is known, and the emissivity is $\sim 1$, the emitted spectrum can be calculated and so the spectrometer and window combination can be calibrated. This calibration can then be used to correct the scans made at other temperatures.

The ratio of corrected signals at any two wavelengths can be used to calculate a temperature. For the wavelengths here at about $1 \mu \mathrm{m}$ or less and at temperatures $1500-3000 \mathrm{~K}$ the Wien approximation can be calculated to be accurate to better than $0.05 \%$ of radiance. Using this approximation gives the ratio of signals, $R$, at temperature $T$ as

$$
R=\frac{I_{1}}{I_{2}}=\left(\frac{\lambda_{2}}{\lambda_{1}}\right)^{5} e^{\frac{c_{2}}{T}\left(\frac{1}{\lambda_{1}}-\frac{1}{\lambda_{2}}\right)}
$$

where $\mathrm{c}_{2}$ is the second radiation constant, $I$ is radiance, $\lambda$ is wavelength and $T$ is thermodynamic temperature The temperature is then given by

$$
T=\frac{-c_{2}\left(\frac{1}{\lambda_{1}}-\frac{1}{\lambda_{2}}\right)}{\operatorname{Ln}\left[\left(\frac{\lambda_{1}}{\lambda_{2}}\right)^{5} \cdot R\right]}
$$

noting that since $R$ tends to 1 as $\lambda_{1}$ tends to $\lambda_{2}$, both numerator and denominator tend to zero as $\lambda_{1}$ approaches $\lambda_{2}$.

The Wien approximation can similarly be written in terms of two temperatures and one wavelength to give

$$
\frac{1}{T_{1}}=\frac{1}{T_{2}}-\frac{\lambda}{c_{2}} \operatorname{Ln}(R)
$$

\subsection{Pyrometer measurements}

Secondly, a radiation thermometer (Land Instruments S1, developed as part of Himert project [7]) was used, again viewing through the furnace window and between the induction coil, to measure all three fixed points. Although the thermometer was calibrated and had the raw signal output available, it was treated as a "temperature display only" device. In this case the first step is to estimate a wavelength. It has been shown [4] that the emissivity control (where present) can be used to interrogate a radiation thermometer and evaluate its wavelength response. In a similar way, a beam splitter or neutral filter of known transmittance can be used to attenuate the signal by a known amount, which allows an estimate of an effective wavelength. The results given in [5] can be written as

$$
f\left(T_{x}, \lambda_{0}, \sigma\right)=\frac{A}{S_{x}}
$$

where $A$ is a constant, $S_{x}$ is the measured signal and

$$
f\left(T_{x}, \lambda_{0}, \sigma\right)=\exp \left[\frac{c_{2}}{\lambda_{0}\left(1-\frac{6 \sigma^{2}}{\lambda_{0}^{2}}\right) T_{x}+\frac{c_{2} \sigma^{2}}{2 \lambda_{0}^{2}}}\right]
$$

$\lambda_{0}$ is close to the centre wavelength and $\sigma$ depends on the filter shape and is typically in the range of fwhm/4 fwhm/2. $f\left(T, \lambda_{0}, \sigma\right)$ is only weakly dependent on $\sigma$ and an estimated value may suffice for any approximately bandpass filter response.

We can write

$$
f\left(T_{1}, \lambda_{0}, \sigma\right)=\frac{S_{2}}{S_{1}} f\left(T_{2}, \lambda_{0}, \sigma\right)
$$

and so assess the effect of a change in signal ratio $S_{2} / S_{1}$ on the indicated temperatures $T_{1}$ and $T_{2}$. Assuming some knowledge of the filter characteristics, a value for $\sigma$ is estimated. For example for a narrow band interference filter $10 \mathrm{~nm}$ to $25 \mathrm{~nm}$ fwhm, a value of $\sigma$ from $5 \mathrm{~nm}$ to $10 \mathrm{~nm}$ would be appropriate. For a broadband filter a value of $\sigma$ from $20 \mathrm{~nm}$ to $50 \mathrm{~nm}$. Any value for $\lambda_{0}$ can be set. Reducing the signal by a known amount, for example using a neutral filter of known transmittance, will reduce the indicated temperature from $T_{1}$ to $T_{2}$. If the chosen value for $\lambda_{0}$ is correct then $T_{2}$ calculated from (7) will agree with the measured change. It is then just a matter of iterating to an ever better $\lambda_{0}$ value such that the correct change in temperature is found. Having evaluated the radiation thermometer, a measurement of an in-situ fixed point can now be fully evaluated. The embedded fixed point is measured through the furnace window and the indicated melting temperature becomes $T_{2}$, while the known reference temperature is $T_{l}$. With values for $\lambda_{0}$, $\sigma, T_{1}$ (the known fixed point temperature) and $T_{2}$ (the fixed point temperature as measured through the window) $S_{2} / S_{I}$ can now be evaluated as the transmission, $\tau$, of the window. Substituting (6) into (7) now allows a corrected temperature $T_{1}$ to be calculated at any arbitrary temperature $T_{2}$. The value of $\sigma$ can now be varied to assess the possible error introduced by an incorrect initial guess, and an allowance made in the uncertainty budget.

To test this approach the measurements made using the $\mathrm{S} 1$ pyrometer with high temperature fixed points in the VITI furnace were analysed. Since the raw output signal was known in this case, it was simple to simulate a reduction in signal to test the method of assessing the wavelength. The analysis was carried out using the ruthenium-carbon fixed point as reference to determine a wavelength and window correction, and the corrections were checked against the cobalt-carbon and rhenium-carbon fixed points.

To further check the method a "polka dot" beamsplitter (a partially coated reflective patterned substrate giving uniform transmittance regardless of wavelength or angle of use) with a measured transmission factor of $42 \%$ was used to set the ratio $S_{I} / S_{2}$. Using a variable temperature blackbody as a source, and a partially metallised window from an ebeam furnace, measurements were made of the open furnace, the furnace with just the window and the furnace with both window and beam splitter. The thermometer used had a narrow band interference filter with a centre wavelength of $650 \mathrm{~nm}$ and fwhm of 10 
$\mathrm{nm}$. In the analysis a value of $25 \mathrm{~nm}$ as an estimate for the fwhm was used initially (more suited to a broadband instrument), then $5 \mathrm{~nm}$ to test the uncertainties.

\section{Results}

\subsection{Spectra measurement}

The first step is to identify the scan closest to the reference temperature: this can be done by taking a slice through Figure 2 at any wavelength and identifying the scan closest to the point of inflection [8].

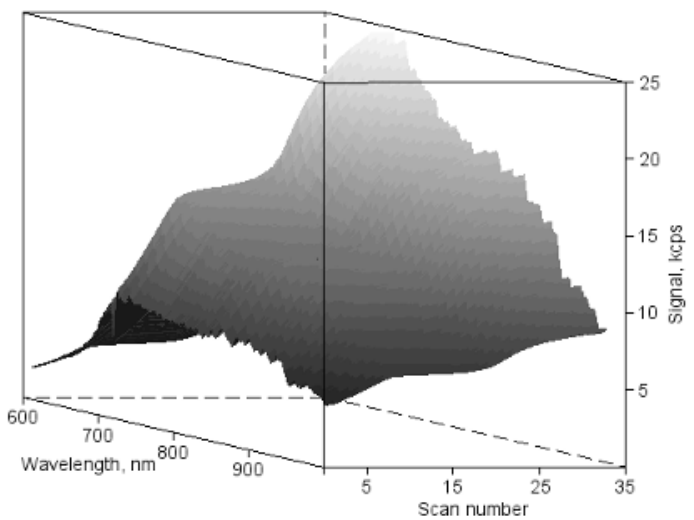

Figure 2. successive spectra taken while a ruthenium-carbon fixed point is heated through its melting transition. The signal at any wavelength is the combined effect of a blackbody spectra and the spectrometer response, in this case peaking between $700 \mathrm{~nm}$ and $800 \mathrm{~nm}$. The spectra flatten off as melting progressses and the point of inflection at any wavelength - here at scan number 15 - is taken as the melting temperature. The signal increases at later scans as the fixed point stabilises at the higher furnace set point temperature.

A calibration can be determined by comparing this scanned spectrum to a blackbody at the known reference temperature of the fixed point, in this case assigning $2226 \mathrm{~K}$ to scan \#15 in Figure 2, with the result shown in Figure 3.

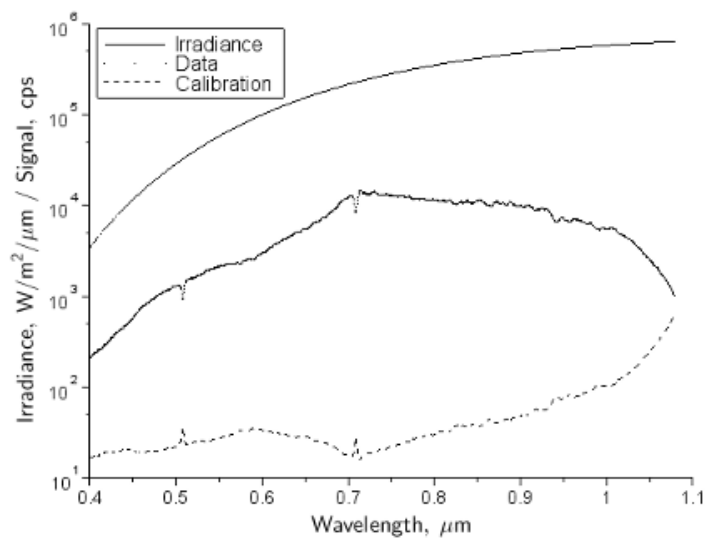

Figure 3. By comparing the spectrometer signal at the ruthenium-carbon melting curve point of inflection to the calculated irradiance of a blackbody at the same temperature, a calibration curve can be determined.
The spectra from scans at lower and higher temperatures, measurements made as the fixed point is heated through its phase transition, can then be corrected using this calibration curve. Then the ratio $R$ from equation (2) can be calculated for any pair of wavelengths $\lambda_{1}$ and $\lambda_{2}$, and equation (3) used to calculate the corresponding temperature. Some typical results are shown in Figure 4.

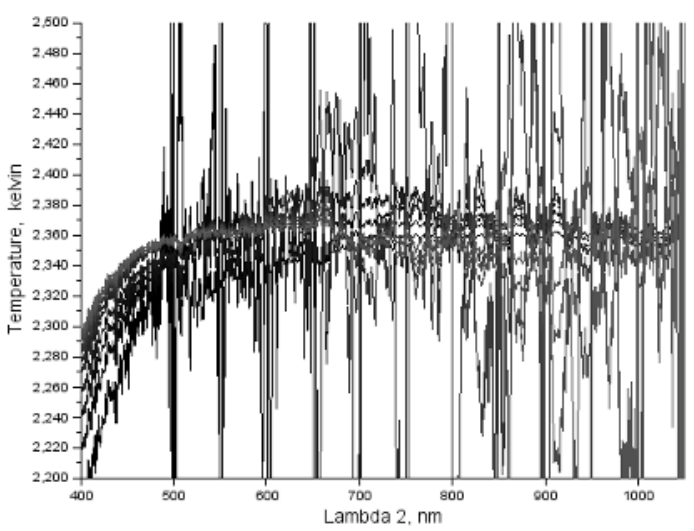

Figure 4. Overlaid plots of temperature calculated from the ratio of wavelengths for $\lambda_{1}$ from $500 \mathrm{~nm}$ to $1050 \mathrm{~nm}$ at $50 \mathrm{~nm}$ steps for a single scanned spectrum (\#33). Spikes occur every $50 \mathrm{~nm}$ at $\lambda_{1}=\lambda_{2}$. Data is for scan 33 from Figure 2.

This shows the temperature calculated for a single scan (\#33 - the highest temperature after melting observed in Figure 2). Selected values of $\lambda_{1}$ at $50 \mathrm{~nm}$ intervals from $500 \mathrm{~nm}$ to $1050 \mathrm{~nm}$ are plotted for $\lambda_{2}$ from $400 \mathrm{~nm}$ to $1050 \mathrm{~nm}$. There is a fall off at shorter wavelength, especially below $500 \mathrm{~nm}$. Spikes appear where $\lambda_{1}=\lambda_{2}$ and equation (3) is not valid. Provided data is restricted to $\lambda_{1,2}>600 \mathrm{~nm}$ and care is taken when $\lambda_{1}$ is close to $\lambda_{2}$ the results are very consistent at a temperature of about $2350 \mathrm{~K}$.

Using the high temperature fixed point reference temperature $T_{2}=2226 \mathrm{~K}$ in equation (4), the spectra from each scan (\#1 to \#33) can be analysed. The results are shown in Figure 5. Here the results cluster together where the measured fixed point is passing through the melting transition defined to be $2226 \mathrm{~K}$. As with equation (3) plotted in Figure 4, there is a fall off at wavelengths below about $600 \mathrm{~nm}$. 


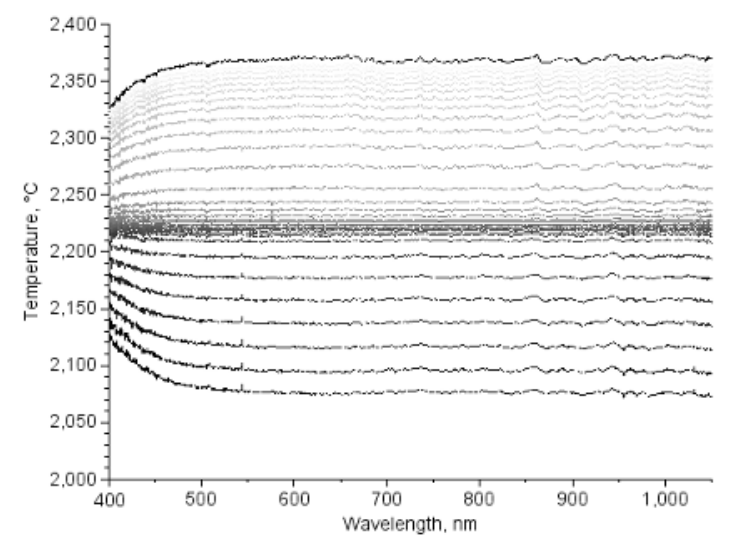

Figure 5. spectral temperature calculated relative to reference at $2226 \mathrm{~K}$ for the 33 consecutive scans from Figure 1. The scans compress around the melting temperature of he fixed point.

To assess the differences the results from equations (3) and (4) were compared by taking the data $600 \mathrm{~nm}$ to $1000 \mathrm{~nm}$. For equation (4) this simply amounts to averaging the data for each line plotted in Figure 5. For equation (3), a single line is taken from Figure 4, corresponding to one determination of temperature. In this case $1050 \mathrm{~nm}$ was used as $\lambda_{2}$ in equation (3), and the average of results for $\lambda_{1}$ from $600 \mathrm{~nm}$ to $900 \mathrm{~nm}$ (avoiding $\lambda_{1}=\lambda_{2}$ ). This is repeated for each of the 33 scans. The results are in Figure 6 . The same plot shows the standard deviation of each dataset. The two equations agree well, but using equation (3) (equivalent to a ratio thermometer) has higher scatter in the spectral temperature value. As would be expected, the melting plateau is apparent and the standard deviation is reduced near the reference temperature.

\subsection{Transmission measurement}

The ruthenium fixed point was taken to have a melting temperature of $2226 \mathrm{~K}$. The S1 pyrometer measuring through the window gave a value of $2194 \mathrm{~K}$. Since this instrument has been calibrated and the raw signal is available it is a simple matter to simulate the effect of halving the signal intensity and to calculate what should be seen if the signal were reduced with a neutral filter or beam splitter. In this case halving the signal should have meant the S1 would read $1990 \mathrm{~K}$ viewing the ruthenium-carbon fixed point though the furnace window.

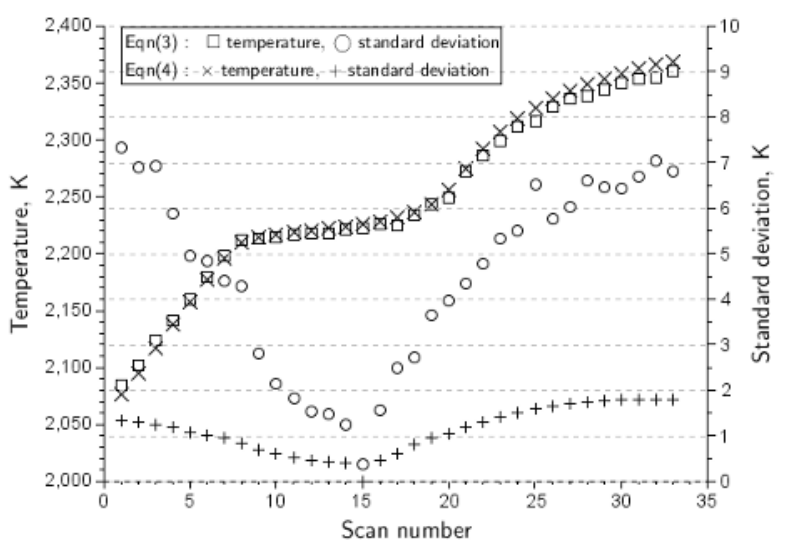

Figure 6: Temperatures determined using equations (3) and (4), with a reference of $2226 \mathrm{~K}$ at scan 15 . Equation (3) uses a fixed wavelength at $1050 \mathrm{~nm}$ and then the average temperature and standard deviation from $600 \mathrm{~nm}$ to $900 \mathrm{~nm}$ is used. Eqn (4) is the average and standard deviation from $600 \mathrm{~nm}$ to $1000 \mathrm{~nm}$.

The spectral response bandwidth was estimated as the $\mathrm{S} 1$ is known to have a cut on filter at nominally $865 \mathrm{~nm}$ and at the long wavelength is limited by the silicon photodiode detector response. A value of $\sigma=$ $25 \mathrm{~nm}$ was used. Making successively better approximations using eqn (7) gives a wavelength $\lambda_{0}$ of $971 \mathrm{~nm}$. Since the parameters in the right hand side of eqn (6) are now known, eqn (7) can be used to determine the window transmission as 0.908 using the known and measured fixed point values. With $\lambda_{0}, \sigma$ and transmission $S_{1} / S_{2}$ known, corrections at the other fixed points can be evaluated. Correcting the measured temperatures of cobalt-carbon and rhenium-carbon used in the VITI furnace gives values of $1323.5^{\circ} \mathrm{C}$ for cobalt-carbon and $2476.5^{\circ} \mathrm{C}$ for rhenium-carbon. These are in good agreement with published values at $1324{ }^{\circ} \mathrm{C}$ and $2474{ }^{\circ} \mathrm{C}$ respectively.

Measurements were made under fully controlled conditions to validate these findings. The LP3 radiation thermometer viewed a Thermogauge variable temperature blackbody source. At $250{ }^{\circ} \mathrm{C}$ intervals from $1000{ }^{\circ} \mathrm{C}$ to $2500{ }^{\circ} \mathrm{C}$ the blackbody temperature and LP3 signal output were recorded, then the measurement was repeated with a "polka dot" beam splitter, and then repeated again with a partly metallised window of unknown transmission together with the beamsplitter. The change in signal with the beam splitter gives its transmission at $44.2 \%$. This value could, of course, have been determined independently, or an absorptive neutral filter of known optical density used. The measured temperatures at one of the blackbody settings (nominal $1750{ }^{\circ} \mathrm{C}$, close to the known platinum-carbon high temperature fixed point) were treated as a calibration point. The indicated temperature through the window was $1987.3 \mathrm{~K}$, and with a beam splitter added $1851.65 \mathrm{~K}$. An initial value of $750 \mathrm{~nm}$ for $\lambda_{0}$ was used with an estimated $25 \mathrm{~nm}$ for $\sigma$ (likely to be a gross overestimate: the filter fwhm is about $10 \mathrm{~nm}$ and $\sigma$ will be about 3-5 $\mathrm{nm}$ [4]). These values would require that 
the beamsplitter transmission was $49.5 \%$. By successively changing $\lambda_{0}$ a wavelength of $644.2 \mathrm{~nm}$ was arrived at to give the known $44.2 \%$ transmission. The furnace without either window or beamsplitter had a measured temperature of $2020.3 \mathrm{~K}$. This is enough information to determine a correction curve. Figure 7 shows the correction curve compared to the measured temperature offsets due to the unknown window transmission.

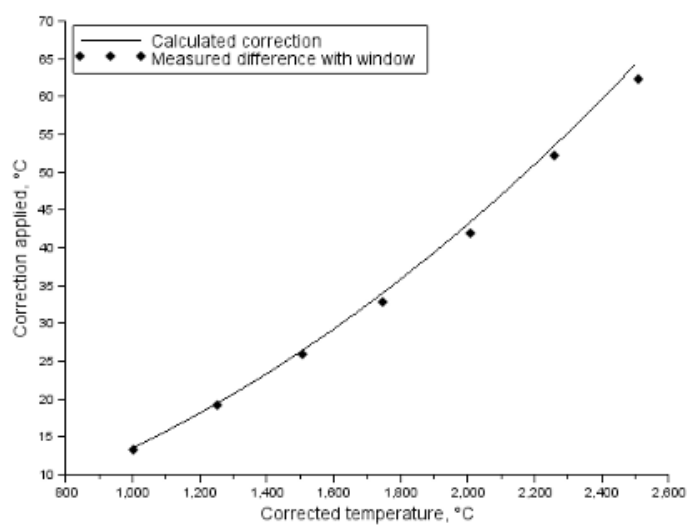

Figure 7. Comparison of the correction curve for the window of unknown transmission, calculated with an assumed $\sigma$ value of $25 \mathrm{~nm}$, compared to the measured temperature difference.

The value of $\sigma$ was deliberately chosen to be wrong $25 \mathrm{~nm}$ would correspond to a broadband spectral response rather than the narrowband used. Since the filter shape and width are known a much better $\sigma$ value of $3 \mathrm{~nm}$ was used and the difference to $\sigma=25 \mathrm{~nm}$ is plotted in Figure 8.

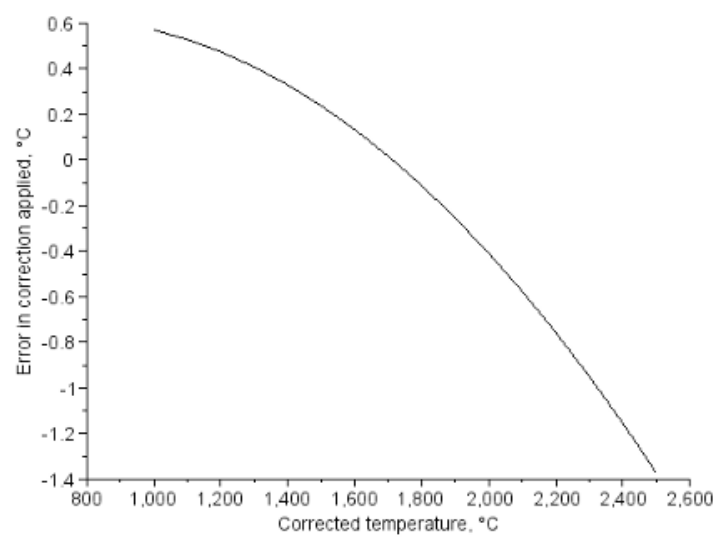

Figure 8 The difference in correction for the LP3 radiation thermometer using an assumed $\sigma=25 \mathrm{~nm}$ (corresponding to a broadband instrument) rather than the more appropriate $3 \mathrm{~nm}$ value

The error from $\sigma$ choice and the difference between calculated and measured correction would be significant for precision measurement at a national measurement institute, but for an industrial environment in difficult conditions through a contaminated window an error of $<5^{\circ} \mathrm{C}$ at $2500{ }^{\circ} \mathrm{C}$ is almost negligible.

\section{Conclusions}

The use of a fixed point in process has been shown to give reduced temperature uncertainties within difficult environments such as measuring through a window. The fixed point reference allows improved measurement knowledge and a better assessment of uncertainties for both ratio and single band radiation thermometers. Only two measurements are required: the fixed point itself and a known reduction in intensity are sufficient to allow a correction to be applied for window transmission even when all the user has is a temperature indicating thermometer. It was shown that a single spot pyrometer gave better results than the simulated two colour device.

\section{Acknowledgements}

This work is part of the High temperature metrology for industrial applications $\left(>1000^{\circ} \mathrm{C}\right)$ project. The HiTeMS project is funded by the European Metrology Research Programme and EMRP is jointly funded by the EMRP participating countries within EURAMET and the European Union.

C.Parga work has been performed thanks to a $\mathrm{PhD}$ grant from CEA.

Thanks to Paul Miller for the loan of the spectrometer and to Gavin Sutton for his advice on setting it up and analysing the results.

The NPL authors acknowledge funding from the National Measurement Office, an Executive Agency of the UK Department of Business, Innovation and Skill.

\section{References}

[1] G. Machin, "Twelve years of high temperature fixed point research: a review" in Temperature : Its Measurement and Control in Science and Industry, vol. 8, edited by C. Meyer, in press, Anaheim (2013)

[2] G. Machin, J. Pearce, Veulban, Strnad, K. Anhalt and M. Sadli, "Progress report for European project $<<$ High temperature metrology for industrial applications $>>$ " in these proceedings

[3] M. Sadli and F. Bourson, "Construction and insitu characterisation of high-temperature fixed point cells devoted to industrial applications" in these proceedings

[4] P. Coates, Metrologia,. 17, 103 (1981)

[5] C. J. Parga, C. Journaeu and A. Tokuhiro, High Temperature High Pressure, 41, 423 (2012)

[6] P. Saunders and D. R. White, Metrologia,40, 195 (2003)

[7] P. Saunders, "Simple methods for characterising the spectral responsivity of radiation thermometers" in Proceedings of Tempmeko 04, Cavtat, Dubrovnik (2004)

[8] G. Machin, G. Beynon, S. Fourrez, J. Hartmann, D. Lowe, R. Morice, M. Sadli and M. Villamanan, 
"HIMERT: a pan-European project for the development of metal-carbon eutectics as temperature standards" in Proceedings of Temperature its Measurement and Control in Science and Industry, Vol. 7, ed. Ripple D., AIP Conference Proceedings, Chicago (2003. 